\title{
Endlich: Leitlinie für Cerumen-Entfernung
}

\section{Hier steht eine Anzeige.}

\section{Wie konnten wir früher bloß ohne Leitlinien durchs Leben kommen? Jetzt gibt es eine für das Manage- ment von Patienten mit Ohrenschmalzpfröpfen.}

() Yurchello108 / Getty Images / istock
_ Ein hochkarätiges Expertengremium formulierte auf Basis intensiver Literaturrecherche zehn Punkte für Diagnostik, Therapie und Management von Patienten mit verstopften Ohren wegen impaktierten Cerumens:

1 Der Arzt sollte den Patienten über die Pflege des Gehörgangs aufklären, um Ceruminalpfröpfe zu vermeiden.

2 Ein Ceruminalpfropf ist zu diagnostizieren, wenn er mit dem Otoskop zu sehen ist, das Ohr deswegen nicht untersucht werden kann und der Patient Symptome hat.

3 Der Arzt sollte nach Antikoagulation, Immunsuppression, Diabetes mellitus, früheren Bestrahlungen im Kopf- und Halsbereich, einer Verengung des Gehörgangs, Exostosen sowie Verletzungen des Trommelfells fragen.

4 Asymptomatische Patienten, deren Ohren ausreichend untersucht werden können, müssen nicht behandelt werden.

5 Bei Kindern und Patienten mit kognitiver Einschränkung, die Symptome nicht adäquat beschreiben können, sollte man die Notwendigkeit einer Intervention anders prüfen.

6 Patienten mit Hörgeräten sollten otoskopisch auf das Vorliegen von Cerumen untersucht werden.

7 Empfohlene Therapien sind die Anwendung von auflösenden Substanzen (z. B. Wasserstoffperoxid, Natriumbicarbonat, Kochsalz, Olivenöl), eine Ohrspülung mit Wasser sowie der Einsatz von Instrumenten.

8 Ärzte sollten von Wattestäbchen abraten.

9 Die Reinigung des Gehörgangs sollte dokumentiert werden. Gelingt die Entfernung des Pfropfs nicht, so sollte an alternative Diagnosen gedacht werden.

10 Gelingt die Erstbehandlung nicht, sollte man an einen Kollegen mit mehr Erfahrung überweisen.

- Schwartz SR et al. Clinical practice guideline (Update): Earwax (Cerumen Impaction). Otolaryngol Head Neck Surg. 2017;156:14-29

\section{KOMMENTAR}

Für die Formulierung dieser bahnbrechenden Empfehlungen auf 29 Druckseiten mit 121 Literaturangaben haben sich 14 renommierte Kollegen an einem Ort in den USA getroffen. An manchen Stellen fühlte ich mich an meine Grundausbildung bei der Bundeswehr erinnert, wo es im Leitfaden für den Soldaten hieß: „Bei Nacht ist mit Dunkelheit zu rechnen."

Prof. Dr. med. H. S. FüeßI 